\title{
Impacto de la educación virtual bajo la pandemia de covid-19 en el proceso de enseñanza-aprendizaje en unidades educativas interculturales bilingües del Ecuador
}

\author{
Luis Marcial Agualongo Chela \\ lagualongo@ueb.edu.ec \\ https://orcid.org/0000-0003-4804-0522 \\ Universidad Estatal de Bolívar, Ecuador \\ Verónica Teresa Veloz Segura \\ vveloz@ueb.edu.ec \\ https://orcid.org/0000-0002-1440-0115 \\ Universidad Estatal de Bolívar, Ecuador \\ Carlos Manuel Núñez Michuy \\ cnunez@ueb.edu.ec \\ https://orcid.org/0000-0003-2298-7697 \\ Universidad Estatal de Bolívar, Ecuador \\ Jhony Patricio Velasco Velasco \\ patricio.velasco@educacion.gob.ec \\ https://orcid.org/0000-0003-1740-2084 \\ Unidad Educativa Ángel Polibio Chaves, Ecuador
}

\section{Universidad Estatal de Bolívar \\ Guaranda - Ecuador}

\section{RESUMEN}

La Educación Intercultural Bilingüe surge de las necesidades, intereses y aspiraciones de las diversas nacionalidades y pueblos indígenas, de conservar sus características propias a través de la educación, pero la pandemia del COVID-19 que enfrenta el mundo, ha provocado cambios en el paradigma educativo, en donde la educación intercultural bilingüe también se ve afectada, este trabajo tiene un enfoque cuantitativo de tipo exploratorio-descriptivo, se seleccionaron dos Unidades Educativas Interculturales Bilingües, una en la Provincia de Bolívar y otra en la Provincia de Loja, para evaluar el efecto de la educación virtual en el proceso de enseñanza aprendizaje y en la aplicación 
del currículo de Educación Intercultural Bilingüe, con la aplicación de una encuesta validada a una muestra de estudiantes, docentes y padres de familia; a pesar de estar las instituciones educativas en provincias tan distantes, presentan problemas similares por falta de conectividad continua y segura, así como también falta de conocimiento en el manejo de herramientas tecnológicas; la mayoría de los padres encuestados, aunque desconfían de la eficacia de las clases virtuales, se han adaptado a esta nueva forma de educación, haciendo inversiones en equipos tecnológicos, y en tiempo junto a sus hijos; la mayoría de los estudiantes muestran confianza en las estrategias metodológicas y contenidos aplicados; los docentes dedican tiempo y esfuerzo en la preparación, aplicación , monitoreo y seguimiento del proceso académico que llevan los estudiantes, alcanzando solo a aplicar los contenidos mínimos del currículo de EIB.

Palabras clave: educación virtual; educación intercultural bilingüe; enseñanzaaprendizaje bilingüe. 


\title{
Impact of virtual education under the covid-19 pandemic in the teaching-learning process in bilingual intercultural educational units in Ecuador
}

\begin{abstract}
Bilingual Intercultural Education arises from the needs, interests and aspirations of the various nationalities and indigenous peoples, to preserve their own characteristics through education, but the COVID-19 pandemic that the world is facing has caused changes in the educational paradigm, where bilingual intercultural education is also affected, this work has a quantitative approach of an exploratory-descriptive type, two Bilingual Intercultural Educational Units were selected, one in the Province of Bolívar and another in the Province of Loja, to evaluate the effect of virtual education in the teaching-learning process and in the application of the Bilingual Intercultural Education curriculum, with the application of a validated survey to a sample of students, teachers and parents; Despite being educational institutions in such distant provinces, they present similar problems due to lack of continuous and secure connectivity, as well as lack of knowledge in handling technological tools; Most of the parents surveyed, although they distrust the effectiveness of virtual classes, have adapted to this new form of education, making investments in technological equipment, and in time with their children; most of the students show confidence in the methodological strategies and applied contents; Teachers dedicate time and effort to the preparation, application, monitoring and follow-up of the academic process that students carry out, reaching only to apply the minimum contents of the EIB curriculum.
\end{abstract}

Keywords: virtual education; bilingual intercultural education; bilingual teachinglearning.

Artículo recibido: 15 enero 2022 Aceptado para publicación: 08 febrero 2022 Correspondencia: lagualongo@ueb.edu.ec Conflictos de Interés: Ninguna que declarar 


\section{INTRODUCCIÓN}

El contexto actual bajo la amenaza de la pandemia por COVID-19 está provocando una serie de transformaciones en las distintas esferas de la vida social, política, laboral y económica. La enseñanza es entendida actualmente como un proceso de ayuda a la construcción que llevan a cabo los docentes (Huerta, 2020).

Los diferentes gobiernos han lanzado iniciativas de política de emergencia basadas en la suspensión de clases y el cierre de centros educativos, para continuar la actividad docente desde los hogares de forma telemática a través del uso de tecnologías de la información y la comunicación para poder detener el número de infecciones (Zhang et al., 2020). Esto ha generado un cambio de paradigma en la educación, que está asumiendo la transferencia de la enseñanza presencial a la enseñanza en línea. Peralta (2015) la educación en línea es una oportunidad no solo de motivar, sino también de guiar y acompañar a los estudiantes en sus proyectos y evaluaciones es decir cómo los profesionales de la educación deben afrontar la adaptación al nuevo contexto global a través de la modalidad online, un movimiento que ha ocurrido y está ocurriendo de manera frenética y abrupta. Parra (2012) asegura que la tecnología ha tenido una gran influencia a nivel educativo siendo una herramienta útil en los procesos de aprendizaje y se ha convertido en parte de la "cotidianidad escolar"

Camino y Maure (2020) nos señalan que la entrega de recursos tecnológicos y un buen uso educativo de los mismos promovería la reducción de las diferencias entre la escuela y sociedad.

Según Maradiaga (2020) menciona que la educación virtual y su impacto es de conocimiento público, ya que estamos inmersos en el contexto de la transformación de las tecnologías de la información y de la comunicación y es un hecho concreto que como producto del negativo impacto de la pandemia COVID-19, la utilización de la tecnología se ha convertido en un imperativo categórico y por ello su funcionalidad y efectividad ha adquirido un dimensionamiento de primera, en el nivel de competitividad será más alto a medida que se utilicen más las tecnologías de la información y comunicación para proyectos, tareas y ejercicios en clase (Paredes, 2016).

Cifuentes (2014) menciona que la educación virtual es una opción y una forma de aprendizaje que se acopla y la necesidad del estudiante y también facilita el manejo de la información y de los contenidos del tema que se desea tratar. 
En consecuencia, los profesionales de la educación están llevando a cabo un proceso de asimilación hacia nuevas circunstancias con algunas dificultades, provocando situaciones de alto nivel de estrés (Espino-Díaz et al., 2020). En el Ecuador, el Sistema de Educación Intercultural Bilingüe de las nacionalidades y pueblos indígenas, comprende desde la estimulación temprana hasta el nivel superior. Está destinado a la implementación del Estado plurinacional e intercultural, en el marco de un desarrollo sostenible con visión de largo plazo (Asamblea Nacional, 2008). El Modelo del Sistema de Educación Intercultural Bilingüe (MOSEIB)(Educación, 2014) es un modelo educativo práctico, que parte de las necesidades, intereses y aspiraciones de las diversas nacionalidades y pueblos indígenas. Está basado en el derecho a ser reconocidos como grupos con características propias conforme a sus cosmovisiones respectivas, considera las formas peculiares de vida, promueve la valoración y recuperación de la cultura de sus ancestros, y contribuye al fortalecimiento social de las respectivas culturas y del país en general, a través de procesos de socialización, descentralización y autonomía; lo cual cuenta con la participación activa en el hecho educativo de los padres de familia, líderes comunitarios, docentes, discentes y organismos nacionales e internacionales (Quishpe C., 2001). Se ha encontrado que los hablantes de lenguas ancestrales, que en su mayoría viven en zonas rurales, suelen estar situados en líneas de pobreza extrema y acuden como tendencia el abandono de sus raíces culturales, para solucionar la precaria situación en que viven (Paronyan \& Cuenca, 2018). A medida que las sociedades occidentales se enfrentan a un crecimiento cultural, lingüístico y diversidad socioeconómica, abordar esta diversidad se convierte en la realidad cotidiana de la población activa de la educación. Para Granados (2015), los recursos tecnológicos se han convertido en recursos educativos para facilitar el aprendizaje rompiendo los medios tradicionales de aprendizaje y brindando aportes asumiendo nuevos retos en la labor de enseñanza y por ende en el proceso de aprendizaje. Aunque la necesidad de profesores interculturalmente competentes es cada vez más reconocida, muchos profesores todavía se sienten mal preparados para trabajar con niños culturales y lingüísticamente diversos (Romijn et al., 2021). En Ecuador, solo el 37 por ciento de los hogares tiene acceso a internet, lo que significa que 6 de cada 10 niños, no pueden continuar con sus estudios a través de plataformas digitales. La situación es más grave para los niños de zonas rurales, donde solo el 16 por ciento de los hogares tiene este servicio (Vohlonen, 2020). Una limitante para el correcto desarrollo de los procesos 
educativos virtuales en este período de pandemia, es la disponibilidad de recursos tecnológicos y el acceso a internet, lo cual es una limitación prestarle una atención adecuada, debido a que afecta la interacción y el intercambio pedagógico (Jordan et al., 2021). Es por esto que la gente "ha tenido que arreglárselas de distintas maneras para continuar con las clases".

Bajo estas nuevas circunstancias se presenta este trabajo de enfoque cuantitativo de tipo exploratorio-descriptivo, donde se evalúa la educación intercultural bilingüe en el Ecuador bajo la pandemia de COVID-19, en dos Unidades Educativa bilingües, una en la Provincia Bolívar y otra en la Provincia de Loja en Ecuador, por medio de una encuesta a padres, docentes y estudiantes, identificando los factores que impactan en la educación virtual y en el proceso de enseñanza-aprendizaje bajo la pandemia de COVID-19.

\section{ESTRATEGIAS METODOLÓGICAS O MATERIALES Y MÉTODOS}

La investigación se desarrolla bajo un enfoque cuantitativo de tipo exploratoriodescriptivo. En la recolección de la información se emplea la técnica de la encuesta y como instrumento una encuesta conformada por cuatro preguntas de tipo dicotómica con respuestas de si o no, que fue validada por un experto antes de su aplicación. Las preguntas responden a siete elementos fundamentales: confianza en las clases virtuales, disponibilidad de dispositivos electrónicos, habilidades con el uso de las herramientas tecnológicas, preparación docente, conectividad con la plataforma de tele formación, contenidos de los cursos y recursos educativos empleados que fortalezcan el proceso de enseñanza aprendizaje. La investigación se llevó a cabo en la Unidad Educativa Telmo Jiménez en la Comunidad Quindigua Central de Guaranda de la Provincia Bolívar, que forma en Educación Inicial y Educación General y Básica (EGB) y cuenta con 142 estudiantes y 9 docentes; y la Unidad Educativa Mushuk Rimak en la parroquia San Lucas de la Provincia de Loja, que forma en Educación Inicial, EGB y Bachillerato General Unificado (BGU) y cuenta con 388 estudiantes y 25 docentes; el cuestionario se aplica con una muestra en cada Unidad Educativa, la cual está conformada por 50 estudiantes de últimos cursos de cada unidad educativa, todos los docentes y por 50 padres de familia. El análisis cuantitativo de los resultados se resumió mediante la estadística descriptiva y el análisis de frecuencia absoluta. Una vez obtenidas las respuestas se procedió a su procesamiento mediante el tabulador electrónico Excel determinando los porcentajes obtenidos. 


\section{RESULTADOS Y DISCUSIÓN}

Se aplicó la encuesta de forma virtual a los padres, estudiantes y docentes. Tomando nota y procesando en Excel; las preguntas y las respuestas en forma porcentual se presentan en la Tabla I.

Tabla I. Preguntas y respuestas de las encuestas

\begin{tabular}{|c|c|c|c|c|c|}
\hline \multirow{3}{*}{ Objetivo } & \multirow{3}{*}{ Preguntas } & \multicolumn{4}{|c|}{ Respuestas } \\
\hline & & \multicolumn{2}{|c|}{ Bolívar } & \multicolumn{2}{|c|}{ Loja } \\
\hline & & $\begin{array}{c}\mathrm{Si} \\
(\%)\end{array}$ & $\begin{array}{l}\text { No } \\
(\%)\end{array}$ & $\begin{array}{l}\mathbf{S i} \\
(\%)\end{array}$ & $\begin{array}{l}\text { No } \\
(\%)\end{array}$ \\
\hline \multirow{4}{*}{ 1.Padres } & $\begin{array}{l}\text { 1.1. ¿En su hogar dispone de herramientas } \\
\text { tecnológicas? (Computador, Teléfono, } \\
\text { Tablet) }\end{array}$ & 41 & 59 & 38 & 62 \\
\hline & $\begin{array}{l}\text { 1.2. ¿Encontraron dificultades en el manejo } \\
\text { de las clases virtuales de su hijo/a? }\end{array}$ & 69 & 31 & 72 & 28 \\
\hline & $\begin{array}{l}\text { 1.3. ¿Si no tiene conectividad, ustedes se } \\
\text { contactan con el docente para usar otros } \\
\text { recursos? }\end{array}$ & 100 & 0 & 100 & 0 \\
\hline & $\begin{array}{l}\text { 1.4. ¿Considera usted que los conocimientos } \\
\text { se transfieren mejor en las clases virtuales } \\
\text { que en las presenciales? }\end{array}$ & 38 & 62 & 15 & 85 \\
\hline \multirow{4}{*}{ 2.Estudiantes } & $\begin{array}{l}\text { 2.1. ¿Considera que los docentes de su unidad } \\
\text { educativa, se encuentran preparados para } \\
\text { enfrentar estos nuevos retos de la educación } \\
\text { virtual? }\end{array}$ & 73 & 27 & 80 & 20 \\
\hline & $\begin{array}{l}\text { 2.2. ¿Existe una conectividad constante en la } \\
\text { unidad educativa, que permita recibir las } \\
\text { clases sin problemas? }\end{array}$ & 20 & 80 & 15 & 85 \\
\hline & $\begin{array}{l}\text { 2.3. ¿Te sientes satisfecho con las estrategias y } \\
\text { recursos empleados por los docentes en tus } \\
\text { clases virtuales? }\end{array}$ & 60 & 40 & 55 & 45 \\
\hline & $\begin{array}{l}\text { 2.4. ¿Consideras que en las clases virtuales se } \\
\text { está aplicando adecuadamente el currículo } \\
\text { de EIB? }\end{array}$ & 60 & 40 & 55 & 45 \\
\hline \multirow{4}{*}{ 3.Docentes } & $\begin{array}{l}\text { 3.1. ¿Existe colaboración mutua entre } \\
\text { estudiante-docente durante el desarrollo de } \\
\text { las clases virtuales? }\end{array}$ & 64 & 36 & 58 & 42 \\
\hline & $\begin{array}{l}\text { 3.2. ¿Aplica los contenidos priorizados del } \\
\text { currículo de EIB? }\end{array}$ & 67 & 33 & 67 & 33 \\
\hline & $\begin{array}{l}\text { 3.3. ¿Existe conectividad en el establecimiento } \\
\text { educativo al servicio de los estudiantes } \\
\text { antes, durante y después de las clases } \\
\text { virtuales? }\end{array}$ & 55 & 45 & 65 & 35 \\
\hline & $\begin{array}{l}\text { 3.4. ¿Cree usted que los contenidos del } \\
\text { currículo de EIB están acorde a la situación } \\
\text { actual? }\end{array}$ & 67 & 33 & 65 & 35 \\
\hline
\end{tabular}




\section{Al analizar las respuestas de las encuestas, encontramos que:}

\section{Los Padres:}

- La mayoría de los padres de familia encuestados indican que no disponen de las herramientas tecnológicas en sus hogares para las clases virtuales, pero han tenido que adaptarse a esta situación.

- La mayoría encontraron dificultades en el manejo de las clases virtuales de sus hijos.

- Todos los padres en caso de problemas de conectividad, se contactan con el docente para que usen otros recursos educativos.

- La mayoría de los padres mencionan que los conocimientos se transfieren mejor en las clases presenciales.

Para Ramírez (2020) en un estudio sobre la percepción de los estudiantes frente a la educación virtual, menciona que, no son buenas debido a varios factores que deben superarse, entre los que se debe destacar lo económico, la deficiente conectividad, las metodologías y medios de enseñanza.

Entonces la mayoría de los padres se han visto en dificultades, porque no contaban con herramientas tecnológicas en sus hogares para que sus hijos participen en las clases virtuales y en muchos casos obligados hacer esfuerzos económicos para suplir de estos dispositivos; así como también fue difícil para la familia el proceso de adaptación para recibir estas clases; en muchas ocasiones se pierde la conectividad, teniendo que comunicarse con los docentes para que les hagan llegar la información y los deberes, por correo o a las redes sociales; la mayoría consideran que en las clases virtuales no aprenden tanto como en las presenciales, por lo que una gran parte de los padres los acompañan durante las clases y la realización de los deberes, como forma de apoyo.

\section{Los Estudiantes:}

- La mayoría de los estudiantes consideran que los docentes están preparados para las clases virtuales.

- La mayoría indican que en su unidad educativa no se cuenta con una conectividad constante, lo que les causa problemas al momento de recibir las clases.

- La mayoría manifiestan que están satisfechos con las estrategias y recursos tecnológicos que aplican los docentes durante las clases virtuales, aunque una parte no lo están.

- La mayoría consideran que los docentes aplican adecuadamente el currículo de 
educación intercultural bilingüe durante las clases virtuales.

La mayoría de los estudiantes consideran que los docentes están preparados para las clases virtuales y que aplican adecuadamente el currículo de EIB; que las unidades educativas no cuentan con una conectividad constante que les permita recibir las clases sin problemas, por lo que piden que se hagan más inversiones en estas; un alto número de estudiantes se sienten satisfechos con las estrategias y recursos tecnológicos que emplean los docentes en sus clases virtuales.

\section{Los Docentes:}

- La mayoría consideran que hay colaboración entre estudiante-docentes durante las clases virtuales.

- La mayoría afirma aplicar los contenidos priorizados o mínimos del currículo de EIB.

- Afirman que en la unidad educativa se mantiene una conectividad para atender a los estudiantes antes, durante y después de las clases virtuales.

- La mayoría consideran que los contenidos del currículo de EIB están acorde a la situación actual.

Los docentes encuestados, consideran que la relación con los estudiantes es buena y que se mantiene una colaboración mutua durante las clases, así como también una predisposición para atender a los estudiantes antes, durante y después de las clases virtuales; en cuanto al currículo de EIB afirman dictar los contenidos mínimos y que estén acorde a la realidad actual del país.

A consecuencia de la pandemia de COVID-19, la educación en todos los niveles en nuestro planeta, ha sufrido un cambio de paradigma, debiendo adaptarse de la modalidad presencial a la virtual. Según (Global,2020) la educación virtual es uno de los nuevos métodos de enseñanza en la actualidad, utiliza la tecnología para la educación a distancia, eliminando las barreras de la distancia y por qué no hay barrera de tiempo. Este proceso ha traído consigo muchos desafíos al proceso de enseñanza y aprendizaje, que incluyen, desde la infraestructura tecnológica de las instituciones y hogares que en ambos casos es deficiente para una conexión estable, hasta un alto nivel de confianza en los docentes y su esfuerzo en la preparación, aplicación , monitoreo y seguimiento de los procesos académicos, así como la dedicación de los estudiantes y apoyo de los padres; la carencia de tecnologías digitales influye en la dedicación de los estudiantes al estudio y en su 
rendimiento académico, por lo que es crucial, que los docentes se adapten a las nueva circunstancias personales y académicas (Pérez López et al., 2020). Además, se debe tener en cuenta que todo esto va unido a la angustia provocada por la pandemia.

\section{CONCLUSIÓN O CONSIDERACIONES FINALES}

- La conectividad continua y segura al servicio de internet y la falta de herramientas tecnológicas, son los dos principales problemas encontrados.

- Los estudiantes confían en las estrategias metodológicas, empleados por los docentes, en el proceso de enseñanza aprendizaje.

- La mayoría de padres, aunque desconfían de la eficacia de las clases virtuales, se han adaptado a esta nueva forma de educación, haciendo inversiones en dispositivos tecnológicos, así como también en tiempo ya que acompañan a sus hijos durante las clases y la realización de deberes, para poder estar seguros de su participación y apoyarlos en caso de algún inconveniente.

- Los docentes dedican tiempo y esfuerzos en la preparación, aplicación, monitoreo y seguimiento de los estudiantes; alcanzando aplicar los contenidos mínimos del currículo de EIB.

- Se podrán crear nuevas metodologías, nuevas actividades didácticas, nuevos currículos, pero mientras se siga pensando en la EIB como un modelo únicamente para indígenas, continuará el contexto de desvaloración de los pueblos indígenas, de su lengua y cultura, sin darle la debida importancia y valor.

\section{LISTA DE REFERENCIAS}

Asamblea Nacional. (2008). Constitución 2008. Incluye Las Reformas Aprobadas En El Referéndum y Consulta Popular de 7 de Mayo Del 2011, Constitución de la República del Ecuador, 1-216. Obtenido de: https://www.asambleanacional.gob.ec/sites/default/files/documents/old/constituc ion_de_bolsillo.pdf

Camino, J. C., \& Maure, R. (2020). Uso de las TICS y su relación con las competencias digitales de los docentes de la institución educativa José Caruana, distrito de Cayma, Arequipa, 2019. [Tesis Licenciatura] Universidad Nacional de San Agustín de Arequipa. http://repositorio.unsa.edu.pe/handle/20.500.12773/11609

Cifuentes, W. M. (25 de Octubre de 2014). Educacion Virtual. Obtenido de Educacion Virtual: https://prezi.com/hwyidupght4t/educacion-virtual/ 
Educación, M. de. (2014). MOSEIB. Modelo del Sistema de Educacion Intercultural Bilingue. Obtenido de: https://educacion.gob.ec/wpcontent/uploads/downloads/2014/03/MOSEIB.pdf

Espino-Díaz, L., Fernandez-Caminero, G., Hernandez-Lloret, C. M., Gonzalez-Gonzalez, H., \& Alvarez-Castillo, J. L. (2020). Analyzing the impact of COVID-19 on education professionals. Toward a paradigm shift: ICT and neuroeducation as a binomial of action. Sustainability (Switzerland), 12(14), 1-10. https://doi.org/10.3390/su12145646

Global. (2020). ¿Que es la educacion virtual? . Obtenido de ¿Que es la educacion virtual?: https://edu.gcfglobal.org/es/educacion-virtual/que-es-la-educacion-virtual/1/

Granados, A. Las TIC en la enseñanza de los métodos numéricos. Sophia Educación, v. 11, n. 2, p. 143-154, 2015.

Huerta, M. (2020). ¿Que es la enseñanza? Revista Internacional Magisterio , 23-24.

Jordan, K., David, R., Phillips, T., \& Pellini, A. (2021). Education during the COVID-19: crisis Opportunities and constraints of using EdTech in low-income countries. Revista de Educación a Distancia (RED), 21(65), 1-15. https://doi.org/10.6018/red.453621

Maradiaga, J. R. (9 de Julio de 2020). La educacion virtual y su impacto . Obtenido de https://www.latribuna.hn/2020/07/09/la-educacion-virtual-y-su-impacto/

Paredes, M. (27 de junio de 2016). definiciones. Obtenido de http://www.scielo.org.mx/scielo.php?script=sci_arttext\&pid=S2007$74672019000200005 \& \operatorname{lng}$

Paronyan, H., \& Cuenca, M. (2018). perfeccionamiento y sostenibilidad Intercultural bilingual education in Ecuador: challenges, development and sustainability Introducción. Transformación, 14(3), 310-326. Obtenido de: http://scielo.sld.cu/pdf/trf/v14n3/2077-2955-trf-14-03-310.pdf

Parra, C. TIC, conocimiento, educación y competencias tecnológicas en la formación de maestros. Revista Nómadas, Bogotá (Colombia), n. 36, p. 145-159, 2012.

Peralta (2015). La plataforma virtual como herramienta de enseñanza. Revista Vinculando.

Pérez López, E., Vázquez Atochero, A., \& Cambero Rivero, S. (2020). Educación a distancia en tiempos de COVID-19: Análisis desde la perspectiva de los 
estudiantes universitarios. RIED. Revista Iberoamericana de Educación a Distancia, 24(1), 331. https://doi.org/10.5944/ried.24.1.27855

Quishpe C. (2001). Educación Intercultural y Bilingüe. Publicación Mensual Del Instituto Científico de Culturas Indígenas., 3(31). Obtenido de: http://icci.nativeweb.org/boletin/31/quishpe.html

Ramírez I. (2020). Percepciones universitarias sobre la educación virtual. Red de docentes IB, 3 (1), 1-6

Romijn, B. R., Slot, P. L., \& Leseman, P. P. M. (2021). Increasing teachers' intercultural competences in teacher preparation programs and through professional development: A review. Teaching and Teacher Education, 98, 103236. https://doi.org/10.1016/j.tate.2020.103236

Vohlonen, A. (2020). COVID-19: Cómo asegurar el aprendizaje de los niños sin acceso a Internet. UNICEF Ecuador, Obtenido de: Https://Www.Unicef.Org/Ecuador/Historias/Covid-19-C\%C3\%B3mo-AsegurarEl-Aprendizaje-de-Los-Ni\%C3\%Blos-Sin-Acceso-Internet.

Zhang, W., Wang, Y., Yang, L., \& Wang, C. (2020). Suspending Classes Without Stopping Learning: China's Education Emergency Management Policy in the COVID-19 Outbreak. Journal of Risk and Financial Management, 13(3), 55. https://doi.org/10.3390/jrfm13030055 